\title{
Trabalho da Equipe Multiprofissional no contexto da COVID-19: Diversos olhares, um só objetivo
}

The Multi-professional Work Team in the context of COVID-19: Several overview, just one purpose

Trabajo del Equipo Multiprofesional en el contexto de COVID-19: Varias Miradas, un propósito

Recebido: 01/03/2021 | Revisado: 09/03/2021 | Aceito: 18/03/2021 | Publicado: 25/03/2021

Itacely Marinho da Silva ORCID: https://orcid.org/0000-0002-0851-1687 Secretaria de Estado da Saúde da Paraíba, Brasil E-mail:itacelymarinho@ hotmail.com

Maria Thayná Bernardo Ferreira da Silva ORCID: https://orcid.org/0000-0002-4069-484X Secretaria de Estado da Saúde da Paraíba, Brasil E-mail: mariaathayna@gmail.com

Robson Gomes dos Santos ORCID: https://orcid.org/0000-0001-8052-8662 Secretaria de Estado da Saúde da Paraíba, Brasil E-mail:robgomes05@outlook.com

Rossana Karla Gois Ferreira ORCID: https://orcid.org/0000-0001-8541-7929 Secretaria de Estado da Saúde da Paraíba, Brasil

E-mail: rossanagoisf@gmail.com

\begin{abstract}
Resumo
A COVID-19 é uma doença causada pelo vírus SARS-CoV-2. O primeiro caso dessa doença foi relatado em Wuhan, na China. Rapidamente o vírus se espalhou ocasionando uma pandemia mundial. A conduta terapêutica para essa infecção requer um olhar ampliado, que considere as consequências fisiológicas e sociais da doença, sendo, portanto, necessário o apoio de uma equipe composta por diversos profissionais da saúde que atuem de forma articulada e colaborativa. Esse trabalho tem como objetivo identificar a literatura científica que trata sobre o trabalho da equipe multiprofissional frente à pandemia. Foi realizada uma revisão integrativa da literatura no período de abril a agosto de 2020. Foram identificados 28 artigos compatíveis com o objetivo deste estudo, predominantemente encontrados na base de dados PubMed, realizados no Brasil e publicados em 2020. São evidentes os diversos impactos na saúde ocasionados por essa doença, exigindo, por conseguinte, uma abordagem multiprofissional. O trabalho multiprofissional favorece uma assistência à saúde integral e contribui para a eficácia dos serviços de saúde nesse tempo de pandemia. A literatura científica traz achados que refletem a contribuição da equipe multiprofissional nesse contexto.
\end{abstract}

Palavras-chave: Infecção por coronavírus; Equipe de saúde; Equipe multiprofissional.

\begin{abstract}
COVID-19 is a disease caused by the SARS-CoV-2 virus. The first case of this disease was reported in Wuhan, China. The virus quickly spread causing a worldwide pandemic. The therapeutic approach for this infection requires an expanded look, which considers the physiological and social consequences of the disease, therefore, it is necessary the support of a team composed of several health professionals that act in an articulate and collaborative way. This work aims to identify the scientific literature that deals with the work of the multidisciplinary team in the face of the pandemic. An integrative literature review was carried out from April to August 2020. It was identified twenty-eight articles compatible with the objective of this study, predominantly found in the PubMed database, carried out in Brazil and published in 2020. The several health impacts caused by this disease are evident, thus requiring a multiprofessional approach. Multi-professional work favors comprehensive health care and contributes to the effectiveness of health services in this time of pandemic. The scientific literature brings findings that reflect the contribution of the multidisciplinary team in this context.
\end{abstract}

Keywords: Coronavirus infections; Health team; Multiprofessional team.

\section{Resumen}

COVID-19 es una enfermedad causada por el virus SARS-CoV-2. El primer caso de esta enfermedad se informó en Wuhan, China. El virus se propagó rápidamente provocando una pandemia mundial. El abordaje terapéutico de esta infección requiere una mirada ampliada, que considere las consecuencias fisiológicas y sociales de la enfermedad, por 
lo que es necesario apoyar un equipo compuesto por varios profesionales de la salud que actúen de manera articulada y colaborativa. Este trabajo tiene como objetivo identificar la literatura científica que aborda el trabajo del equipo multidisciplinario ante la pandemia. Se realizó una revisión integradora de la literatura de abril a agosto de 2020.Se identificaron 28 artículos compatibles con el objetivo de este estudio, predominantemente encontrados en la base de datos PubMed, realizados en Brasil y publicados en 2020. Los diferentes impactos en la salud son evidentes causados por este enfermedad, por lo que requiere un enfoque multiprofesional. El trabajo multiprofesional favorece la atención integral de la salud y contribuye a la efectividad de los servicios de salud en este momento de pandemia. La literatura científica trae hallazgos que reflejan la contribución del equipe multiprofesional en este contexto.

Palabras clave: Infecciones por coronavirus; Equipo de salud; Equipo multiprofesional.

\section{Introdução}

A COVID-19 é uma doença causada pelo novo coronavírus, denominado SARS-CoV-2. Em pouco tempo essa doença espalhou-se por todo o mundo acometendo uma grande quantidade de pessoas, fato que levou a Organização Mundial da Saúde (OMS) a decretar um quadro de pandemia (Guo, et al., 2020).

As principais manifestações clínicas identificadas no curso da doença são febre, fadiga, tosse seca, falta de ar, dores musculares, dor de cabeça, confusão, dor de garganta, rinorréia, dor no peito, diarreia, náusea e vômito (N. Chen, et al., 2020; D. Wang, et al., 2020). Febre e tosse foram os sintomas dominantes, enquanto os sintomas respiratórios superiores e gastrointestinais eram raros, o que indica diferenças no tropismo viral em relação ao SARS-CoV, MERS-CoV, e influenza (Wang, et al., 2016). O controle da sintomatologia é realizado através de medicamentos, e em casos mais graves é realizada oxigenoterapia (Ministério da Saúde [MS], 2020).

$\mathrm{O}$ trabalho em equipe multiprofissional com foco interdisciplinar favorece a garantia de um dos princípios do Sistema Único de Saúde (SUS), a integralidade, além de corroborar com o conceito ampliado de saúde. Esta equipe é composta por diferentes profissionais de saúde, dentre eles, médicos, enfermeiros, farmacêuticos, odontólogos, fisioterapeutas, nutricionistas, fonoaudiólogos, psicólogos e assistentes sociais (Evangelista, et al., 2016). As diversas especialidades atuam de maneira articulada e colaborativa com a finalidade de prestar uma assistência que compreenda todas as necessidades de saúde do mesmo indivíduo (Peduzzi, et al., 2016).

As características fundamentais para o trabalho em equipe incluem: interação e comunicação entre os membros da equipe; presença de objetivos em comum; responsabilidade compartilhada e promoção da inovação no trabalho em busca de novas formas para atender às necessidades do paciente (Agreli, et al., 2017). O apoio multiprofissional perpassa todos os níveis de assistência, desde a Atenção Primária a Saúde (APS) até os pacientes críticos da Unidade de Terapia Intensiva (UTI), e contribui para uma assistência à saúde integral, humanizada e eficaz (Evangelista, et al., 2016; Peduzzi \& Agreli, 2018).

Considerando os benefícios da equipe multiprofissional no cuidado à saúde torna-se essencial discutir sobre a atuação desse formato de equipe no atual contexto da saúde. Portanto, esse trabalho tem como objetivo identificar na literatura científica as evidências relacionadas ao trabalho da equipe multiprofissional frente à pandemia.

\section{Metodologia}

Trata-se de uma revisão integrativa, na qual sua condução percorreu as seguintes etapas: elaboração da questão de pesquisa; busca na literatura dos estudos primários; extração de dados; avaliação dos estudos primários; análise e síntese dos resultados e apresentação da revisão (Polit \& Beck, 2006). A pesquisa foi orientada a partir da pergunta norteadora: Quais as contribuições do cuidado multiprofissional e interdisciplinar para prevenção, suspeição, tratamento e reabilitação da COVID19 ?

Os critérios de inclusão escolhidos para a seleção das publicações foram: artigos nas categorias originais e revisão de literatura, incluindo revisões sistemáticas, relacionados ao tema e notas técnicas, publicados em língua portuguesa e inglesa. 
As publicações consideradas para o desenvolvimento dessa pesquisa estão entre os anos de 2016 e 2020, considerando um corte temporal que reporta um período que antecipa a identificação do primeiro caso de COVID-19 no mundo, e um período após ( $\mathrm{Li}$, et al., 2020). O uso de notas técnicas na presente investigação se justifica pelo fato de que, o estudo tratar de uma discussão ainda nova no campo da ciência, e a natureza desses documentos traz informações fundamentais no que concerne as medidas de controle e propagação da COVID-19, na sociedade e nos serviços de saúde.

Foram excluídas da pesquisa as publicações que não estiveram relacionadas com o objetivo desta revisão bibliográfica, publicações nos formatos de trabalho de conclusão de curso, dissertação, tese, relato de caso, resenha e resumo de congresso, publicações que não estiveram disponibilizados on-line em formato completo, bem como em idiomas além do português e inglês.

A busca de trabalhos para a pesquisa primária ocorreu entre abril e agosto de 2020. As bases de dados pesquisadas foram: Scientific Electronic Library Online (Scielo), National Library of Medicine (Pubmed), Sciencedirect, Google Scholar, além de publicações do Ministério da Saúde e Organização Mundial da Saúde. A busca foi norteada pelos descritores Infecção por Coronavírus, assistência à saúde, Equipe de Assistência ao Paciente, enfermagem, Nutricionistas, fonoaudiologia, fisioterapia, farmácia e saúde mental, pesquisados em português e inglês, obedecendo aos Descritores em Ciências da Saúde (Decs).

Os descritores foram cruzados entre si, partindo do descritor principal (Infecção por Coronavírus). Fato que torna-se melhor correspondido em informações no Quadro 1.

Quadro 1. Passos realizados para cruzamento dos descritores. João Pessoa - PB, 2020.

\begin{tabular}{|c|c|}
\hline Passos & Cruzamento dos Descritores \\
\hline $1^{\circ}$ & "Infecção por Coronavírus" AND "Assistência à saúde" OR "Equipe de Assistência ao Paciente" \\
\hline $2^{\circ}$ & "Infecção por Coronavírus" AND “Assistência à saúde” AND "Enfermagem” \\
\hline $3^{\circ}$ & "Infecção por Coronavírus" AND “Assistência à saúde” AND "Nutricionistas" \\
\hline $4^{\circ}$ & "Infecção por Coronavírus" AND "Assistência à saúde" AND "Fonoaudiologia" \\
\hline $4^{\circ}$ & "Infecção por Coronavírus" AND “Assistência à saúde” AND "Fisioterapia" \\
\hline $5^{\circ}$ & "Infecção por Coronavírus" AND "Assistência à saúde” AND "Farmácia” \\
\hline $6^{\circ}$ & "Infecção por Coronavírus" AND "Assistência à saúde" AND "Saúde mental” \\
\hline
\end{tabular}

Fonte: Autores.

Utilizando-se os descritores mencionados, foram obtidos 354 artigos. Após leitura dos trabalhos encontrados, participaram da segunda etapa 43 estudos que relacionavam a equipe multiprofissional no cuidado à saúde frente ao contexto da pandemia por covid-19. Nessa fase, excluíram-se 11 artigos por não apresentar texto em seu formato completo e 3 por idioma não correspondente. Portanto, do total de artigos encontrados, 29 foram identificados como compatíveis com o objetivo deste estudo. A seguir descreve-se o fluxograma do processo de seleção dos estudos. 
Research, Society and Development, v. 10, n. 3, e53210313439, 2021

(CC BY 4.0) | ISSN 2525-3409 | DOI: http://dx.doi.org/10.33448/rsd-v10i3.13439

Figura 1. Fluxograma de seleção dos estudos. João Pessoa - PB, 2020.
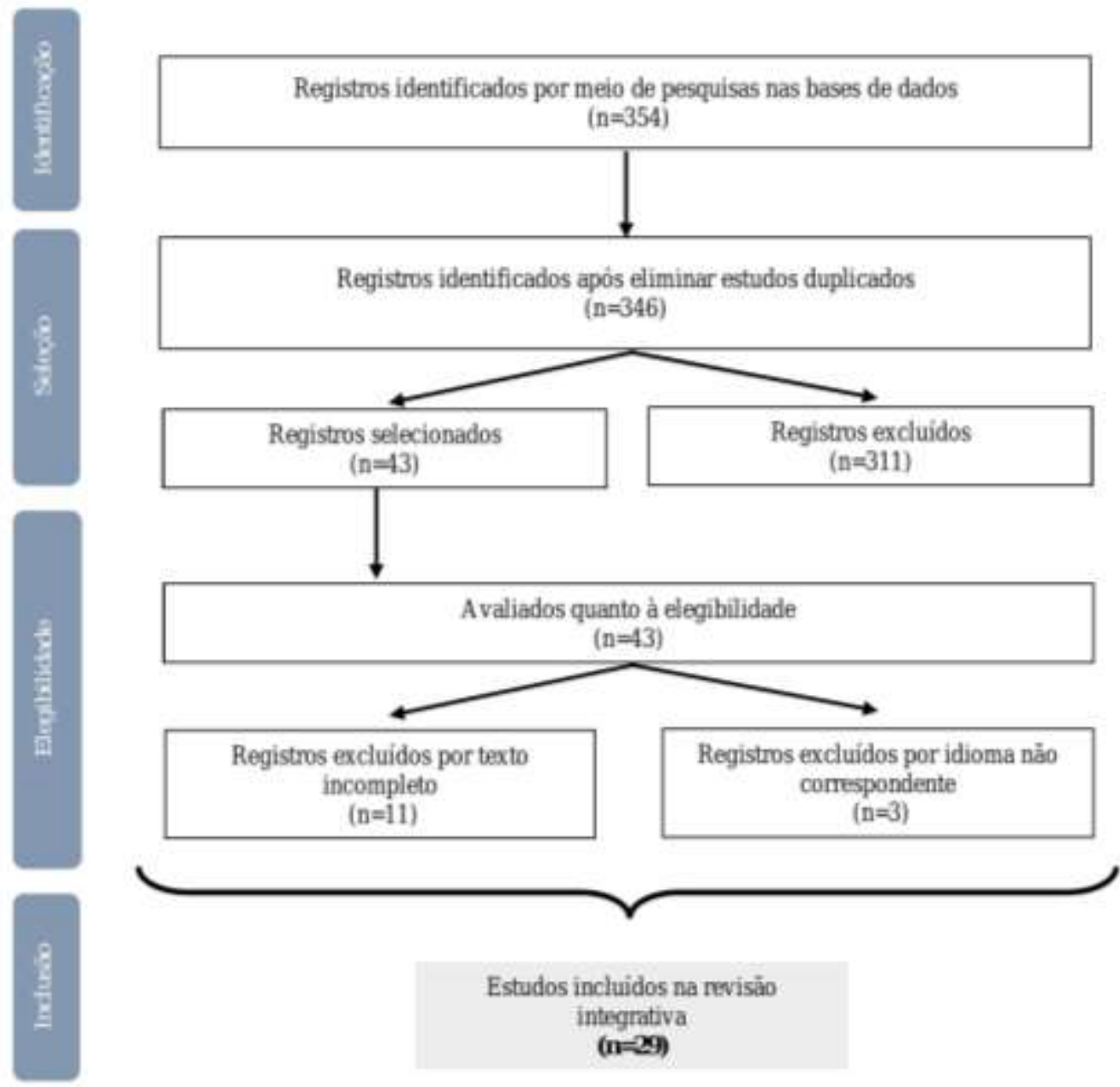

Estudos incluidos na revisăo integrativa $(n=29)$

Fonte: Autores.

\section{Resultados e Discussão}

Em relação à base de dados, os artigos selecionados foram encontrados 15 (53,57\%) na PubMed, 7 (25,00\%) na Scielo, 3 (10,71\%) no Google Scholar, 2 (7,14\%) na ScienceDirect e 2 (7,14\%) publicações do Ministério da Saúde, conforme apresentado na Figura 2.

Figura 2. Artigos distribuídos de acordo com as bases de dados correspondentes.



Fonte: Autores. 
A maioria foi desenvolvida no Brasil 18 (64,28\%), seguido de 7 (25,00\%) na China, 2 (7,14\%) na Espanha, 1 (3,57\%) na Itália e $1(3,57 \%)$ na Irlanda, conforme apresentado na Figura 3 houve predomínio de estudos (90\%) no ano de 2020.

Figura 3. Artigos distribuídos de acordo com o país de realização da pesquisa.

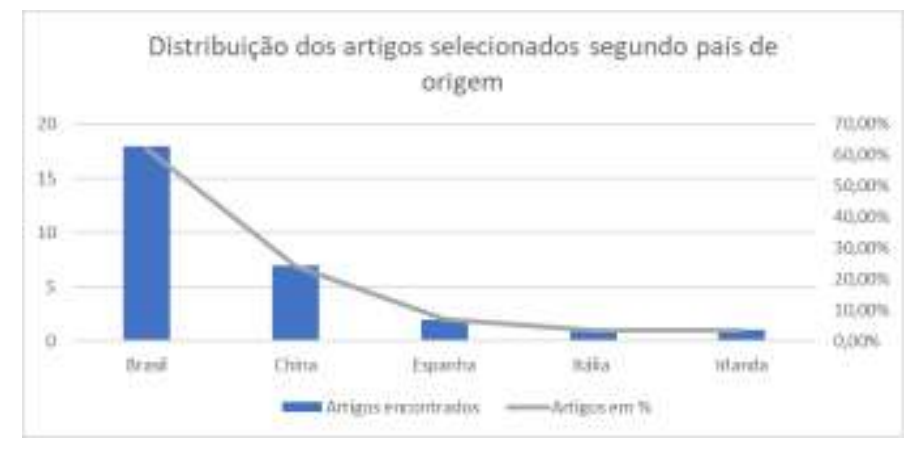

Fonte: Autores.

Os artigos constituintes da amostra, representados por códigos, segundo o título, base de dados em que foram localizados, periódicos em que foram publicados, ano de publicação e país de realização da pesquisa encontram-se descritos no Quadro 2.

Quadro 2. Código do artigo segundo seu título, base de dados, periódico ano e país de origem. João Pessoa - PB, 2020.

\begin{tabular}{|c|c|c|c|c|c|}
\hline COD. & TÍTULO & BASE & PERIÓDICO & ANO & PAÍS \\
\hline A1 & $\begin{array}{l}\text { Clinical features of patients infected } \\
\text { with } 2019 \text { novel coronavirus in } \\
\text { Wuhan, China. }\end{array}$ & PubMed & Lancet & 2020 & China \\
\hline A2 & $\begin{array}{l}\text { Diretrizes para Diagnóstico e } \\
\text { Tratamento da COVID- } 19 .\end{array}$ & $\begin{array}{l}\text { Endereço eletrônico } \\
\text { do Ministério da } \\
\text { Saúde }\end{array}$ & Ministério da Saúde & 2020 & Brasil \\
\hline A3 & $\begin{array}{l}\text { Early transmission dynamics in } \\
\text { Wuhan, China, of novel } \\
\text { coronavirus-Infected pneumonia }\end{array}$ & PubMed & $\begin{array}{l}\text { New England Journal of } \\
\text { Medicine }\end{array}$ & 2020 & China \\
\hline A4 & $\begin{array}{l}\text { Epidemiological and clinical } \\
\text { characteristics of } 99 \text { cases of } 2019 \\
\text { novel coronavirus pneumonia in } \\
\text { Wuhan, China: a descriptive study. }\end{array}$ & PubMed & Lancet & 2020 & China \\
\hline A5 & $\begin{array}{l}\text { Clinical characteristics of } 138 \\
\text { hospitalized patients with } 2019 \\
\text { novel coronavirus-infected } \\
\text { pneumonia in Wuhan, China. }\end{array}$ & ScienceDirect & JAMA & 2020 & China \\
\hline A6 & $\begin{array}{l}\text { Factors associated with clinical } \\
\text { outcome in } 25 \text { patients with avian } \\
\text { influenza a (H7N9) infection in } \\
\text { Guangzhou, China. }\end{array}$ & ScienceDirect & BMC Infect Dis & 2016 & China \\
\hline A7 & $\begin{array}{l}\text { Novel Coronavirus Infection } \\
\text { (COVID-19) in Humans: A } \\
\text { Scoping Review and Meta-Analysis }\end{array}$ & PubMed & J Clin Med & 2020 & Brasil \\
\hline A8 & $\begin{array}{l}\text { O remdesivir e a cloroquina inibem } \\
\text { efetivamente o novo coronavírus } \\
\text { recém-surgido }(2019-n C o V) \text { in } \\
\text { vitro }\end{array}$ & PubMed & Pesquisa Celular & 2020 & Brasil \\
\hline A9 & $\begin{array}{l}\text { A percepção sobre o trabalho em } \\
\text { equipe multiprofissional dos } \\
\text { trabalhadores de um Centro de }\end{array}$ & Scielo & Interface & 2017 & Brasil \\
\hline
\end{tabular}




\begin{tabular}{|c|c|c|c|c|c|}
\hline & $\begin{array}{l}\text { Atenção Psicossocial em Salvador, } \\
\text { Bahia, Brasil }\end{array}$ & & & & \\
\hline A10 & $\begin{array}{l}\text { Sentidos atribuídos à integralidade: } \\
\text { entre o que é preconizado e vivido } \\
\text { na equipe multidisciplinar. }\end{array}$ & Scielo & Interface. & 2017 & Brasil \\
\hline A11 & $\begin{array}{l}\text { Saúde mental dos profissionais de } \\
\text { enfermagem do brasil no contexto } \\
\text { da pandemia covid-19: ação do } \\
\text { conselho federal de enfermagem. }\end{array}$ & Scielo & Cogitare Enfermagem & 2020 & Brasil \\
\hline A12 & $\begin{array}{l}\text { Fatores de estresse nos profissionais } \\
\text { de enfermagem no combate à } \\
\text { pandemia da COVID-19: síntese de } \\
\text { evidências. }\end{array}$ & PubMed & Comun. ciênc. & 2020 & Brasil \\
\hline A13 & $\begin{array}{l}\text { Physiotherapy services in the face } \\
\text { of a pandemic. }\end{array}$ & Scielo & Rev. Assoc. Med. Bras. & 2020 & Brasil \\
\hline A14 & $\begin{array}{l}\text { Atuação do fisioterapeuta em } \\
\text { unidades de terapia intensiva no } \\
\text { contexto da pandemia de COVID- } \\
19 .\end{array}$ & Scielo & Fisioter. mov. & 2020 & Brasil \\
\hline A15 & $\begin{array}{l}\text { Preliminary results of a clinical } \\
\text { study to evaluate the performance } \\
\text { and safety of swallowing in critical } \\
\text { patients with COVID-19 }\end{array}$ & PubMed & Clinics & 2020 & Brasil \\
\hline A16 & $\begin{array}{l}\text { Fonoaudiologia, amamentação e } \\
\text { COVID-19: informações aos } \\
\text { fonoaudiólogos }\end{array}$ & Scielo & CoDas & 2020 & Brasil \\
\hline A17 & $\begin{array}{l}\text { ESPEN expert statements and } \\
\text { practical guidance for nutritional } \\
\text { management of individuals with } \\
\text { SARS-CoV-2 infection }\end{array}$ & PubMed & Clinical Nutrition & 2020 & Itália \\
\hline A18 & $\begin{array}{l}\text { The Inflammation Link and the } \\
\text { Role of Nutrition in Potential } \\
\text { Mitigation }\end{array}$ & PubMed & Nutrients & 2020 & Irlanda \\
\hline A19 & $\begin{array}{l}\text { COVID-19 Pandemic. New } \\
\text { challenge for hospital pharmacy } \\
\text { services. }\end{array}$ & PubMed & Farm Hosp. & 2020 & Espanha \\
\hline A20 & $\begin{array}{l}\text { Hospital Pharmacy in the } \\
\text { multidisciplinary team of COVID } \\
\text { inpatient units. }\end{array}$ & PubMed & Farm Hosp. & 2020 & Espanha \\
\hline A21 & $\begin{array}{l}\text { "Medo pandêmico" e COVID-19: } \\
\text { carga e estratégias de saúde mental }\end{array}$ & PubMed & Jornal Brasileiro de Psiquiatria & 2020 & Brasil \\
\hline A22 & $\begin{array}{l}\text { COVID-19 e saúde mental: a } \\
\text { emergência do cuidado }\end{array}$ & PubMed & Estudos de Psicologia & 2020 & Brasil \\
\hline A23 & $\begin{array}{l}\text { Estratégias para reorganização da } \\
\text { Rede de Atenção à Saúde em } \\
\text { resposta à Pandemia COVID-19: a } \\
\text { experiência do Sistema de Saúde } \\
\text { Italiano na região de Lazio }\end{array}$ & Google Scholar & Aps em Revista & 2020 & Brasil \\
\hline A24 & $\begin{array}{l}\text { Portarias publicadas sobre COVID- } \\
19\end{array}$ & $\begin{array}{l}\text { Endereço eletrônico } \\
\text { do Ministério da } \\
\text { Saúde }\end{array}$ & Ministério da Saúde & 2020 & Brasil \\
\hline A25 & $\begin{array}{l}\text { Ações federais para apoio e } \\
\text { fortalecimento local no combate ao } \\
\text { COVID-19: a Atenção Primária à } \\
\text { Saúde (APS) no assento do } \\
\text { condutor }\end{array}$ & Scielo & Ciênc. saúde coletiva & 2020 & Brasil \\
\hline A26 & $\begin{array}{lll}\text { Vital Surveillances: } & \text { The } \\
\text { Epidemiological } & \text { Characteristics } & \text { of }\end{array}$ & PubMed & China CDC Weekly & 2020 & China \\
\hline
\end{tabular}




\begin{tabular}{|l|l|l|l|l|l|}
\hline & $\begin{array}{l}\text { an Outbreak of 2019 Novel } \\
\text { Coronavirus Diseases (COVID-19) }\end{array}$ & & & \\
\hline A27 & $\begin{array}{l}\text { Enfrentando a COVID-19: APS } \\
\text { forte agora mais que nunca! }\end{array}$ & Google Scholar & Aps em Revista & 2020 & Brasil \\
\hline A28 & $\begin{array}{l}\text { Desafios e recomendações para o } \\
\text { cuidado intensivo de adultos } \\
\text { críticos com doença de coronavírus } \\
2019 \text { (COVID-19) }\end{array}$ & $\begin{array}{l}\text { Revista HRJ (Health Residencies } \\
\text { Journal) }\end{array}$ & 2020 & Brasil \\
\hline
\end{tabular}

Fonte: Autores.

A análise dos dados obtidos possibilitou a definição de quatro categorias: COVID-19: aspectos gerais; Equipe multiprofissional em perspectiva interdisciplinar; Trabalho multiprofissional no tratamento da COVID-19; Assistência em saúde no contexto da pandemia.

\section{COVID-19: Aspectos Gerais}

Sendo uma doença infecciosa respiratória aguda emergente, a doença do SARS-COV-2, responsável pelo atual quadro de pandemia, se espalha principalmente pelo trato respiratório, através de gotículas, secreções respiratórias e contato direto (Li, et al., 2020) A maioria dos adultos ou crianças com infecção por SARS-CoV-2 quando não assintomáticos, apresentam sintomas leves de gripe, entretanto, alguns casos entram em estado crítico desenvolvendo a síndrome do desconforto respiratório agudo, insuficiência respiratória, falência de múltiplos órgãos, podendo evoluir à morte (Huang, et al., 2020).

As principais manifestações clínicas identificadas no curso da doença são febre, fadiga, tosse seca, falta de ar, dores musculares, dor de cabeça, confusão, dor de garganta, rinorréia, dor no peito, diarreia, náusea e vômito (N. Chen, et al., 2020; D. Wang, et al., 2020). Febre e tosse foram os sintomas dominantes, enquanto os sintomas respiratórios superiores e gastrointestinais eram raros, o que indica diferenças no tropismo viral em relação ao SARS-CoV, MERS-CoV, e influenza (Wang, et al., 2016).

Nascimento et al (2020) quantificou a frequência de cada sintoma em mais de 40.000 pacientes. Foi verificado que o sintoma de febre prevaleceu em $82 \%$ deles $(n=4410)$, depois a tosse, com ou sem escarro, em $61 \%(n=3985)$ dos casos. As dores musculares e/ou fadiga foram relatadas em 36\% ( $\mathrm{n}=3778)$, dispneia em 26\% ( $\mathrm{n}=3700)$, dor de cabeça em 12\% ( $\mathrm{n}=3598)$, dor de garganta em 10\% (n=1387) e sintomas gastrointestinais em 9\% ( $\mathrm{n}=1744)$ dos pacientes.

A síndrome do desconforto respiratório agudo (SDRA) é uma complicação grave da COVID-19. O estudo de Huang et al (2020) investigou 138 pacientes e mostrou que a SDRA ocorreu em 20\% dos casos, dos quais 12,3\% exigiram ventilação mecânica.

Por ser uma doença emergente o tratamento ainda não apresenta um padrão com comprovação científica estabelecida e padronizada. Terapias medicamentosas e com plasma convalescente e imunoglobulina $G$ são estudadas, porém sem resultados conclusivos (M. Wang, et al., 2020; L. Chen, et al., 2020). Atualmente o tratamento visa o controle da sintomatologia, através de medicamentos, e garantia do suporte respiratório, quando necessário, através de oxigenoterapia (MS, 2020).

\section{Equipe Multiprofissional em Perspectiva Interdisciplinar}

Segundo Anjos Filho e Souza (2017) o trabalho em equipe constitui-se de uma vinculação mútua de troca técnica e relacional entre os profissionais envolvidos, proporcionando articulação colaborativa e interdisciplinar no serviço prestado ao usuário, de forma que tenha efetividade na intervenção proposta pela prática integral considerando as diversas visões das áreas profissionais que compõe a equipe de saúde. 
Nesse contexto, a interdisciplinaridade dentro do âmbito multiprofissional se expressa como um compartilhamento de mesma base de trabalho procedendo perante uma concepção em comum. Dessa forma, a partir da combinação e troca de saberes, se realizaria o trabalho proposto com postura conjunta e única objetivando o mesmo fim (Anjos Filho \& Souza, 2017), sendo assim utilizada como uma importante estratégia para proporcionar uma assistência de cuidado integralizado ao paciente, refletindo de forma positiva na qualidade da assistência prestada no serviço de saúde (Silva, et al., 2017).

Como já citado no presente estudo os acometimentos no paciente com COVID-19 são diversos e agressivos em alguns casos o que implica na necessidade da intervenção das múltiplas áreas. Isso posto, a equipe multiprofissional mais do que nunca precisa observar e tratar o paciente como um todo, desde questões que perpassam a situação física até circunstâncias emocionais que a internação e o distanciamento social proporcionam.

\section{Trabalho Multiprofissional no Tratamento da COVID-19}

Cada profissional em sua linha de saber é essencial no caminho para solucionar o problema em questão, tanto na área de pesquisa quanto de intervenção direta ao paciente.

A enfermagem se destaca na detecção precoce de casos suspeitos e avaliação dos casos confirmados, não apenas em razão de sua capacidade técnica/científica, mas também por fazerem parte do maior número de profissionais da área da saúde, e serem a única categoria que está nas 24 horas junto ao paciente, assim como, junto à população de forma geral nas diversas linhas do cuidado em saúde (Humerez, et al., 2020). As competências do enfermeiro e de toda equipe de enfermagem também se destaca na aplicação de protocolos estabelecidos pelo Ministério da Saúde relacionados à pandemia. Além da atuação em ações de educação em saúde e divulgação de informações seguras e relevantes a fim de diminuir a contaminação no território em que trabalham, cabendo também colaborar com as medidas de vigilância e controle epidemiológico através da notificação (Barbosa, et al., 2020).

A fadiga intensa, dispneia e sintomas torácicos graves compatíveis com a pneumonia são manifestações clínicas significativas da COVID-19. A piora do quadro na doença caracteriza-se por hipoxemia importante, decorrente de processos fisiopatológicos distintos que vão prejudicar a relação da ventilação-perfusão, que pode levar o paciente com ventilação espontânea a piorar subitamente, necessitando de intubação e ventilação mecânica. O fisioterapeuta irá atuar monitorando e intervindo no contexto de problemas respiratórios agudos, subagudos e crônicos, nos casos de ventilação espontânea, mecânica invasiva e não invasiva, além de proporcionar recuperação da mobilidade e da capacidade funcional dos pacientes, melhorando a qualidade de vida desses (Minghelli, et al., 2020; Guimarães, 2020).

O prolongado tempo de intubação eleva o risco de disfagia pós extubação induzindo a aspiração silente de alimentos e líquidos em direção às vias aéreas inferiores, aumentando as chances de ocorrer uma infecção torácica, pneumonia e desnutrição. Os fonoaudiólogos atuam diretamente na reabilitação da deglutição dos pacientes para garantir uma alimentação oral segura (Lima, et al., 2020). Atua também em neonatos, para certificar a manutenção do aleitamento materno, mesmo no caso de mãe infectada, com toda proteção e adequação necessária (Miranda, et al., 2020). Já o profissional nutricionista atua com o objetivo de manter ou recuperar o estado nutricional do paciente, com foco na prevenção dessa desnutrição, modulação da cascata inflamatória e da imunidade através de nutrientes com potencial anti-inflamatório e imunomodulador (Barazzoni, et al., 2020; Zabetakis, et al., 2020).

A assistência farmacêutica para o tratamento de pacientes acometidos pela COVID-19 inclui monitorar as interações medicamentosas, mantendo a atenção a possíveis reações adversas, observando a necessidade de substituição de medicamentos ou boa interação entre eles para melhora do quadro do paciente e gerenciamento de possíveis riscos. Além disso, a tomada de decisão sobre o tratamento medicamentoso apoiada na avaliação e decisão clínica tem se mostrado fundamental para uma 
prescrição de qualidade e segura no tratamento dos pacientes (Bermejo-Vicedo \& Gorgas, 2020; Gil-Navarro \& LuqueMárquez, 2020).

As consequências psicossociais desse tempo de isolamento social atingem diretamente a saúde mental da população. Ornell et al (2020) e Faro et al (2020) pontuam as emoções negativas, como o medo, a tristeza e a angústia, o afastamento de familiares e amigos, o tédio e a alta carga de ansiedade como fatores contribuintes para o desgaste da saúde mental da população, além de todas as implicações sociais que a pandemia causou como fechamento de escolas e empresas, desempregos, mudanças na rotina de trabalho e organização familiar e sobrecarga dos serviços de saúde.

Diante do exposto, percebemos que o impacto na saúde do indivíduo com COVID-19, seja de maneira leve ou mais grave, exige um olhar multiprofissional, particularmente quando o sistema de saúde busca prover uma assistência integral.

\section{Assistência em Saúde na Pandemia}

Considerando a complexidade do vírus e diante do quadro de pandemia, notoriamente observou-se a necessidade de revisão da prática em saúde em todos os países atingidos e criação de novos protocolos para melhor assistir aos pacientes acometidos pela COVID-19, assim como estratégias para proteger os profissionais que estão na linha de frente. Por exemplo, como traz o estudo de Tasca e Massuda (2020) sobre o sistema de saúde na região de Lazio, em resposta à pandemia, na Itália, houve a implementação de estratégias a partir de um plano de enfrentamento definido conforme os cenários epidemiológicos com fases sistematizadas para possibilitar a reorganização do fluxo, tanto de acesso quanto de atendimento para usuários nãoCOVID e COVID considerando também as fases de evolução da epidemia.

No Brasil, um conjunto de medidas estratégicas foi realizado para o enfrentamento da COVID-19, desde a APS até a UTI, de maneira que objetiva abranger de forma integral toda a assistência multidisciplinar de saúde para os diferentes graus de acometimento dos pacientes (MS, 2020).

O MS estabeleceu, em todos os níveis de assistência, uma rápida resposta reforçando a centralidade da integração das ações de vigilância em saúde com a APS, principalmente devido ao grande número de pessoas que possivelmente apresentarão sintomas leves $(80,9 \%)$ e que, com a finalidade de evitar a propagação do vírus, deverão ser atendidas e direcionadas para tratamento em isolamento domiciliar (Harzheim, et al., 2020; Epidemiology Working Group for NCIP Epidemic Response, Chinese Center for Disease Control and Prevention, 2020).

Dessa forma, nota-se a importância da APS para coordenação, organização e comunicação do cuidado aos pacientes no enfrentamento da doença no Brasil, buscando através do trabalho da equipe multidisciplinar diminuir os encaminhamentos desnecessários e superlotação de hospitais públicos e privados que focariam a atenção em casos mais graves.

Portanto, pode-se dizer que a contribuição fundamental da APS nesse momento é garantir a continuidade de atendimento às pessoas, fortalecer o trabalhar em equipe, informar e alertar a população quanto aos riscos, ainda que necessariamente à distância, para garantir uma atenção à saúde integral (Nedel, 2020).

Na UTI a elevada carga de trabalho dos profissionais envolvidos tem tido influência direta para o aumento da taxa de mortalidade dos pacientes. Neste sentido, todos os profissionais devem ser preparados para atender essa demanda por meio de treinamento específico para COVID-19 e seu gerenciamento dentro da UTI, mesmo que por hora não integrem a equipe mínima atuante, mas como forma de capacitação preventiva, podendo até gerar uma espécie de rodízio de plantões, caso necessário, objetivando melhorar a qualidade da assistência e minimizar os efeitos da situação atual na saúde física e mental dos profissionais da linha de frente (Fernandes, et al., 2020).

Muitas dúvidas e desafios ainda circundam o ambiente de UTI diante da COVID-19 e sobre o comportamento dessa doença nos indivíduos com diferentes condições físicas. Enquanto estudos estão sendo feitos para sanar cada vez mais essa lacuna, a equipe de UTI deve se antecipar aos prováveis desafios associados à pandemia se preparando ao máximo para 
proteger os profissionais de saúde e organizar o serviço de forma a prestar a melhor assistência possível aos pacientes (Fernandes, et al., 2020).

\section{Conclusão}

São evidentes os diversos impactos na saúde ocasionados pela COVID-19, exigindo, portanto, uma abordagem multiprofissional na assistência. Ainda são escassos os artigos que integrem a atuação dos diversos profissionais da saúde e os reflexos da atuação da equipe multiprofissional na assistência à saúde na pandemia. Porém, já é possível identificar na literatura científica a contribuição da equipe multiprofissional nesse contexto.

O atual cenário desafia a organização de trabalho das equipes de saúde. É imprescindível que os gestores e as próprias equipes multiprofissionais valorizem o trabalho de cada especialidade e priorizem a comunicação e a construção de estratégias entre setores e serviços realizados em equipe para garantia de uma assistência à saúde integral e eficaz.

\section{Referências}

Agreli, H. F., Peduzzi, M., \& Bailey, C. (2017). Contributions of team climate in the study of interprofessional collaboration: A conceptual analysis. Journal of interprofessional care, 31(6), 679-684. https://doi.org/10.1080/13561820.2017.1351425

Anjos Filho, N. C. dos, \& Souza, A. M. P. de. (2017). A percepção sobre o trabalho em equipe multiprofissional dos trabalhadores de um Centro de Atenção Psicossocial em Salvador, Bahia, Brasil. Interface - Comunicação, Saúde, Educação, 21(60), 63-76. https://dx.doi.org/10.1590/1807-57622015.0428

Barazzoni, R., Bischoff, S. C., Breda, J., Wickramasinghe, K., Krznaric, Z., Nitzan, D., Pirlich, M., Singer, P., \& endorsed by the ESPEN Council (2020). ESPEN expert statements and practical guidance for nutritional management of individuals with SARS-CoV-2 infection. Clinical nutrition (Edinburgh, Scotland), 39(6), 1631-1638. https://doi.org/10.1016/j.clnu.2020.03.022

Barbosa, D. J., Gomes, M. P., Souza, F. B. A. de, \& Gomes, A. M. T. (2020). Fatores de estresse nos profissionais de enfermagem no combate à pandemia da COVID-19: síntese de evidências. Com. Ciências Saúde, 31(1): 31-47. https://doi.org/10.51723/ccs.v31iSuppl\%201.651

Bermejo-Vicedo, T., \& Gorgas, M. Q. (2020). COVID-19 Pandemic. New challenge for hospital pharmacy services. Pandemia COVID-19. Un nuevo desafío para los servicios de farmacia hospitalaria. Farmacia hospitalaria: organo oficial de expresion cientifica de la Sociedad Espanola de Farmacia Hospitalaria, 44(7), 3-4. http://dx.doi.org/10.7399\%2Ffh.11511

Borges do Nascimento, I. J., Cacic, N., Abdulazeem, H. M., von Groote, T. C., Jayarajah, U., Weerasekara, I., \& Marcolino, M. S. (2020). Novel Coronavirus Infection (COVID-19) in Humans: A Scoping Review and Meta-Analysis. Journal of clinical medicine, 9(4), 941. https://doi.org/10.3390/jcm9040941

Chen, L., Xiong, J., Bao, L., \& Shi, Y. (2020). Convalescent plasma as a potential therapy for COVID-19. The Lancet. Infectious diseases, 20(4), 398-400. https://doi.org/10.1016/S1473-3099(20)30141-9

Chen, N., Zhou, M., Dong, X., Qu, J., Gong, F., Han, Y., \& Zhang, L. (2020). Epidemiological and clinical characteristics of 99 cases of 2019 novel coronavirus pneumonia in Wuhan, China: a descriptive study. Lancet (London, England), 395(10223), 507-513. https://doi.org/10.1016/S0140$6736(20) 30211-7$

Epidemiology Working Group for NCIP Epidemic Response, Chinese Center for Disease Control and Prevention (2020). Zhonghua liu xing bing xue za zhi = Zhonghua liuxingbingxue zazhi, 41(2), 145-151. https://doi.org/10.3760/cma.j.issn.0254-6450.2020.02.003

Evangelista, V. C., Domingos, T. da S., Siqueira, F. P. C., \& Braga, E. M. (2016). Equipe multiprofissional de terapia intensiva: humanização e fragmentação do processo de trabalho. Revista Brasileira de Enfermagem, 69(6), 1099-1107. https://doi.org/10.1590/0034-7167-2016-0221

Faro, A., Bahiano, M. A., Nakano, T. C., Reis, C., Silva, B. F. P. da, \& Vitti, L.S. (2020). COVID-19 e saúde mental: a emergência do cuidado. Estudos de Psicologia (Campinas), 37, e200074. https://doi.org/10.1590/1982-0275202037e200074

Fernandes, C. A., Cunha, A. M. da, Hemkemaier, E. C. R. C., Carmo, K. S. do, Moraes, T. V. P., Santos, T. P. dos, \& Mateus, N. C. (2020). Desafios e recomendações para o cuidado intensivo de adultos críticos com doença de coronavírus 2019 (COVID-19). Health Residencies Journal, 1(1), 1-26. https://doi.org/10.51723/hrj.v1i1.20

Gil-Navarro, M. V., \& Luque-Márquez, R. (2020). Hospital Pharmacy in the multidisciplinary team of COVID inpatient units. La farmacia hospitalaria en el equipo multidisciplinar de las unidades COVID de hospitalización. Farmacia hospitalaria : organo oficial de expresion cientifica de la Sociedad Espanola de Farmacia Hospitalaria, 44(7), 40-42. https://doi.org/10.7399/fh.11517

Guimarães, F. (2020). Atuação do fisioterapeuta em unidades de terapia intensiva no contexto da pandemia de COVID-19. Fisioterapia em Movimento, 33, e0033001. https://doi.org/10.1590/1980-5918.033.ed01

Guo, Y. R., Cao, Q. D., Hong, Z. S., Tan, Y. Y., Chen, S. D., Jin, H. J., \& Yan, Y. (2020). The origin, transmission and clinical therapies on coronavirus disease 2019 (COVID-19) outbreak - an update on the status. Military Medical Research, 7(1), 11. https://doi.org/10.1186/s40779-020-00240-0 
Harzheim, E., Martins, C., Wollmann, L., Pedebos, L. A., Faller, L. de A., Marques, M. das C., Minei, \& D'Avila, O. P. (2020). Ações federais para apoio e fortalecimento local no combate ao COVID-19: a Atenção Primária à Saúde (APS) no assento do condutor. Ciência \& Saúde Coletiva, 25 (1), $2493-2497$. https://doi.org/10.1590/1413-81232020256.1.11492020

Huang, C., Wang, Y., Li, X., Ren, L., Zhao, J., Hu, Y., \& Cao, B. (2020). Clinical features of patients infected with 2019 novel coronavirus in Wuhan, China. Lancet (London, England), 395(10223), 497-506. https://doi.org/10.1016/S0140-6736(20)30183-5

Humerez, D. C. de, Ohl, R. I. B., \& Silva, M. C. N. da. (2020). Saúde mental dos profissionais de enfermagem do Brasil no contexto da pandemia covid-19: ação do Conselho Federal de Enfermagem. Cogitare Enfermagem, 25: e74115. http://dx.doi.org/10.5380/ce.v25i0.74115

Li, Q., Guan, X., Wu, P., Wang, X., Zhou, L., Tong, Y., \& Feng, Z. (2020). Early Transmission Dynamics in Wuhan, China, of Novel Coronavirus-Infected Pneumonia. The New England journal of medicine, 382(13), 1199-1207. https://doi.org/10.1056/NEJMoa2001316

Lima, M. S. de, Sassi, F. C., Medeiros, G. C., Ritto, A. P., \& Andrade, C. R. F. de. (2020). Preliminary results of a clinical study to evaluate the performance and safety of swallowing in critical patients with COVID-19. Clinics, 75: e2021. https://doi.org/10.6061/clinics/2020/e2021

Minghelli, B., Soares, A., Guerreiro, A., Ribeiro, A., Cabrita, C., Vitoria, C., Nunes, C., Martins, C., Gomes, D., Goulart, F., Santos, R. M. dos, \& Antunes, R. (2020). Physiotherapy services in the face of a pandemic. Revista da Associação Médica Brasileira,66(4), 491-497. https://doi.org/10.1590/18069282.66.4.491

Ministério da Saúde. (2020). Diretrizes para Diagnóstico e Tratamento da COVID-19. https://ufsj.edu.br/portal-repositorio/File/lapsam/APA_-_ANPAD.pdf

Ministério da Saúde. (2020). Portarias publicadas sobre COVID-19, Assessoria de Comunicação Social. http://observatoriohospitalar.fio cruz.br/sites/default/files/biblioteca/20.04.2020_Portarias\%20publicadas\%20sobre\%20COVID_com\%20edi\%C3\%A7\%C3\%A3o.pdf

Miranda, V. S. G. de, Rech, R. S., Maahs, M. A. P., Berbert, M. C. B., \& Almeida, S. T. de. (2020). Fonoaudiologia, amamentação e COVID-19: informações aos fonoaudiólogos. CoDAS, 32(3), e20200124. https://doi.org/10.1590/2317-1782/20192020124

Nedel, F. (2020). Enfrentando a COVID-19: APS forte agora mais que nunca!. APS EM REVISTA, 2(1), 11-16. https://doi.org/10.14295/aps.v2i1.68

Ornell, F., Schuch, J. B., Sordi, A. O., \& Kessler, F. (2020). "Pandemic fear" and COVID-19: mental health burden and strategies. Revista brasileira de psiquiatria (Sao Paulo, Brazil: 1999), 42(3), 232-235. https://doi.org/10.1590/1516-4446-2020-0008

Peduzzi, M., \& Agreli, H. F. (2018). Trabalho em equipe e prática colaborativa na Atenção Primária à Saúde. Interface - Comunicação, Saúde, Educação, 22(2), 1525-1534. https://doi.org/10.1590/1807-57622017.0827

Peduzzi, M., Oliveira, M. A. de C., Silva, J. A. M. da, Agreli, H. L. F., \& Miranda Neto, M. V. de. (2016). Trabalho em equipe, prática e educação interprofissional. In Clínica médica: atuação da clínica médica, sinais e sintomas de natureza sistêmica, medicina preventiva, saúde da mulher, envelhecimento e geriatria (Vol. 1). Barueri: Manole.

Polit, D. F; Beck C. T. (2006). Using research in evidence-based nursing practice. In, editors. Essentials of nursing research. Methods, appraisal and utilization. Philadelphia (USA): Lippincott Williams \& Wilkins.

Silva, M. V. S. da, Miranda, G. B. N., \& Andrade, M. A. de. (2017). Sentidos atribuídos à integralidade: entre o que é preconizado e vivido na equipe multidisciplinar. Interface - Comunicação, Saúde, Educação, 21(62), 589-599. https://doi.org/10.1590/1807-57622016.0420

Tasca, R., \& Massuda, A. (2020). Estratégias para reorganização da Rede de Atenção à Saúde em resposta à Pandemia COVID-19: a experiência do Sistema de Saúde Italiano na região de Lazio. APS EM REVISTA, 2(1), 20-27. https://doi.org/10.14295/aps.v2i1.65

Wang, D., Hu, B., Hu, C., Zhu, F., Liu, X., Zhang, J., \& Peng, Z. (2020). Clinical Characteristics of 138 Hospitalized Patients With 2019 Novel CoronavirusInfected Pneumonia in Wuhan, China. JAMA, 323(11), 1061-1069. https://doi.org/10.1001/jama.2020.1585

Wang, H., Xiao, X., Lu, J., Chen, Z., Li, K., Liu, H., \& Yang, Z. (2016). Factors associated with clinical outcome in 25 patients with avian influenza A (H7N9) infection in Guangzhou, China. BMC infectious diseases, 16(1), 534. https://doi.org/10.1186/s12879-016-1840-4

Wang, M., Cao, R., Zhang, L., Yang, X., Liu, J., Xu, M., \& Xiao, G. (2020). Remdesivir and chloroquine effectively inhibit the recently emerged novel coronavirus (2019-nCoV) in vitro. Cell research, 30(3), 269-271. https://doi.org/10.1038/s41422-020-0282-0

Zabetakis, I., Lordan, R., Norton, C., \& Tsoupras, A. (2020). COVID-19: The Inflammation Link and the Role of Nutrition in Potential Mitigation. Nutrients, 12(5), 1466. https://doi.org/10.3390/nu12051466 